\title{
Biodiesel Byproduct and Biofuel Synthesizing Clostridium acetobutylicum
}

\author{
Vincent V. Vuljaj ${ }^{\mathrm{a}}$, James F. Graves ${ }^{\mathrm{a}}$
}

In the manufacture of biodiesel, glycerol is a byproduct. Acetone-butanol-ethanol (ABE) fermentation by anaerobe Clostridium acetobutylicum is also a source of biofuel. Growth of $C$. acetobutylicum in the presence of waste biodiesel glycerol byproduct was examined in this investigation. In cultures containing added glucose or a mixture of glucose and byproduct the optical density (OD) was increased and a positive fermentation reaction was exhibited while results in cultures containing byproduct or pure glycerol were similar to those in the base medium. The $\mathrm{pH}$ of cultures containing glucose markedly decreased. The concentration of glucose dropped to an undetectable level. All cultures were determined to contain alcohol. The potential applications for biodiesel glycerol byproduct need to be explored.

Keywords: Biodiesel, Glycerol, Clostridium acetobutylicum, Butanol

\section{Introduction}

Biodiesel is an alternative fuel substitute for the diesel produced from the petroleum industry. The principal chemical component of biodiesel is fatty acid methyl esters. Biodiesel is commonly made by transesterification, which is a process of alkaline hydrolysis of biological oil by using methanol, sodium hydroxide and heat (http://www.biodiesel.org; http://www.biomich.com; Kalscheuer et al, 2006). Vegetable oils and animal fats provide triacylglycerols, which are the source of fatty acids broken to methyl esters (biodiesel) and glycerol byproduct. Three liters of biodiesel are produced for every liter of glycerol byproduct. Research laboratories are also investigating the use of catalyst in a column to break oil into biodiesel and glycerol (http://www.eng.wayne.edu/page.php?id=4765).

Butanol is a four carbon alcohol that can be used in making of important products such as plasticizers, lacquers and resins. Butanol currently has value for potential use as biofuel (http://www.butanol.com ). Acetone-butanol-ethanol (ABE) microbial fermentation was the main source of butanol and acetone until the early $1950 \mathrm{~s}$ when it became more cost effective to produce these chemicals from the petroleum industry. However, the supply of petroleum is expected to decrease. As the price of gas from petroleum goes up, butanol may be made by the microbial fermentation industry again. Butanol is made by the bacterium known as Clostridium acetobutylicum. It is an anaerobe, which means that it can only grow in the absence of oxygen or it will be killed. Consequently, special environmental conditions are required for culture. The standard feed stock in industry was corn meal mash. Production of butanol by $C$. acetobutylicum from glucose was suggested to be increased in the presence of glycerol (Girbal and Soucaille, 1994; Vasconcelos et al., 1994). Glycerol is not a biofuel but the waste glycerol byproduct from biodiesel plants might be used to make more biofuel.

\section{Materials and methods}

The C. acetobutylicum, catalogue \# 657, was obtained from Presque Isle Cultures, Presque Isle, PA. Biodiesel glycerol byproduct that was made by transesterification was a gift from the biodiesel company, The Power Alternative, Detroit, MI. Byproduct made by an experimental catalyst column was supplied through the generosity of the biofuels laboratory at Next Energy, Wayne State University, Detroit, MI. Brain-heart infusion (BHI), rich in nutrients, was used as a medium because it was expected to be sufficient to support the growth of many types of fastidious bacteria. Fluid thioglycollate medium (FTM) without oxidation reduction (OR) indicator or glucose (manufacturer included glucose) permitted assessment of possible growth from added carbon sources. A characteristic use of FTM is the cultivation of some of the less strict anaerobic bacteria such as clostridia. Sodium thioglycollate in the medium decreased the oxidation reduction potential (Eh) to assist growth of anaerobic bacteria. Phenol red broth base medium was used to screen for fermentation from added carbon sources. If the culture produced acid from added carbon sources, phenol red, the $\mathrm{pH}$ indicator, changed color. The $0.2 \%$ agar was included to enhance the culture of the bacteria. Commercial media was described in detail by Difco (1985). The Oxyrase for broth (Oxyrase, Inc.) enzyme system was used in experiments to assist in creating anaerobiosis. Special commercially prepared enzymes in this supplement protected the anaerobic bacteria from oxygen toxicity. Tube cultures had closed caps and anaerobic plate cultures were incubated in zip-lock bags to help keep out oxygen. The $\mathrm{pH}$ of cultures was determined by spotting samples of culture on $\mathrm{pH}$ paper (Hydrion). The $\mathrm{pH}$ paper permitted the use of a small sample and avoided contamination of a culture with a probe. A pH meter was used for testing of stock solutions and media. Growth of cultures was assessed quantitatively at the end of experiments by vortexing tube contents and measuring optical density (OD), or turbidity, with a Klett - Summerson colorimeter. A rapid solid phase chemistry enzyme (glucose oxidase and peroxidase) based assay (Precision Labs, Inc.) was used to detect glucose in cultures after incubation. A rapid solid phase chemistry enzyme (alcohol oxidase and peroxidase) based assay (AlcoScreen) was used to detect alcohol. The manufacturer suggested that the alcohol test can score for alcohols having up to 4 carbon atoms and will not react with glycerol. In both assays, if the test substrate was present, peroxide produced by the oxidase was used by the peroxidase to alter the color of a dye indicator. 


\section{Results and discussion}

The byproduct from making of biodiesel by standard transesterification was pale yellow and pasty. That from catalyst column was very light and fluid (Figure 1). The byproducts did not appear to be the same substance as glycerol which was clear and syrupy. Other investigators are seeking to research applications for biodiesel glycerol byproduct with the assumption that it is "glycerol" (Murarka et al., 2008). The byproduct may contain other potential nutrient chemicals besides glycerol. Also, many industrial products naturally harbor microbes. Samples $(.1 \mathrm{ml})$ of unsterilized byproduct made by transesterification or catalyst column which were inoculated on $\mathrm{BHI}$ agar, and incubated at $25^{\circ} \mathrm{C}$ for 7 days failed to produce evidence of any resident microbial flora. This suggested that there might not be a need for sterilization of byproduct in some applications in industrial microbiology. Standard media used to grow cultures to make products needs to be sterilized which adds expense to the process. A $20 \%$ stock solution of byproduct made from transesterification showed a high alkaline $\mathrm{pH} 11.0$, in contrast to byproduct from the catalyst column which was acidic with about $\mathrm{pH}$ 4.0. The high alkaline $\mathrm{pH}$ observed with the byproduct from transesterification was likely to be due to the sodium hydroxide used in the manufacturing process. Solutions of glycerol byproduct needed $\mathrm{pH}$ adjustment before use in culture media.

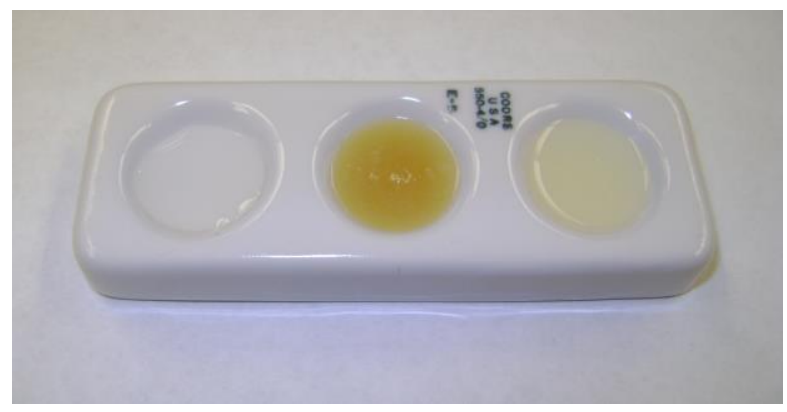

Figure 1. Glycerol byproduct from manufacture of biodiesel. Left, pure glycerol; center,

glycerol byproduct from transesterification; right, glycerol byproduct from catalyst column. Sample size $=0.5 \mathrm{ml}$.

The $C$. acetobutylicum grew well on BHI agar with Oxyrase, in FTM with or without Oxyrase and in BHI and phenol red broth with $0.2 \%$ agar and Oxyrase. The microorganism showed limited growth in BHI with $0.2 \%$ agar. The small amount of agar appeared necessary for culture growth in broth. The organism did not grow well on BHI agar without Oxyrase or in BHI or phenol red broth, with Oxyrase, without the $0.2 \%$ agar. The commercial FTM contained a small amount of agar. Inclusion of byproduct made by transesterification resulted in media with increased turbidity in contrast to that from the catalyst column. Consequently, in addition to a visual evaluation of bacterial growth in experiments, a quantitative assessment by OD was possible with byproduct made from catalyst column as the test substance. In FTM (with Oxyrase) experiment cultures containing added glucose or a mixture of glucose and byproduct from catalyst column culture OD was increased while that in cultures containing byproduct or pure glycerol was similar to that in the base medium (Figure 2). Growth of the microorganism with glucose was not increased by the presence of byproduct. Conversely, the byproduct did not appear to show appreciable toxicity for C. acetobutylicum. For ethanol producing yeast, chemical methods were devised to break down waste biomass (cellulose, hemicellulose and lignin) into digestible subunits (Wyman, 1994: Saha, 2004). However, chemicals toxic to yeast, such as furfural, were produced (Luo, 2002).

In phenol red broth (with $0.2 \%$ agar and Oxyrase) that contained added glucose or a mixture of glucose and byproduct C. acetobutylicum cultures exhibited a positive fermentation reaction in contrast to those containing byproduct or pure glycerol (Figure 3). Fermentation of glucose did not appear to be enhanced or inhibited by the presence of byproduct. Inability of the bacteria to produce a positive reaction in these tests in the presence of the byproduct as the only added supplement suggested that it did not contain a variety of carbon sources for utilization.

\section{$\square$ Optical Density}

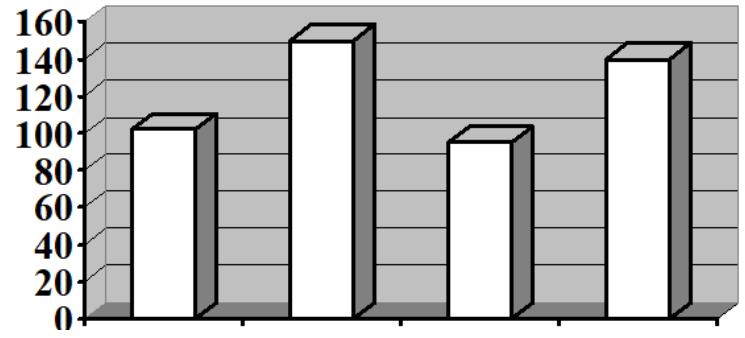

Figure 2. Growth of Clostridium acetobutylicum in fluid thioglycollate medium (with Oxyrase) prepared with biodiesel byproduct. Supplements L to R: none; glucose; byproduct from catalyst column; glucose and byproduct mix (1/2 molar ratio assuming that byproduct was glycerol). Pure glycerol was not shown. Supplements were added at $0.5 \%$ concentration. Inoculum was $0.1 \mathrm{ml}$ of maximum stationary phase cells in $10.0 \mathrm{ml}$ of medium. The incubation was $25^{\circ} \mathrm{C}$ for 6 weeks.

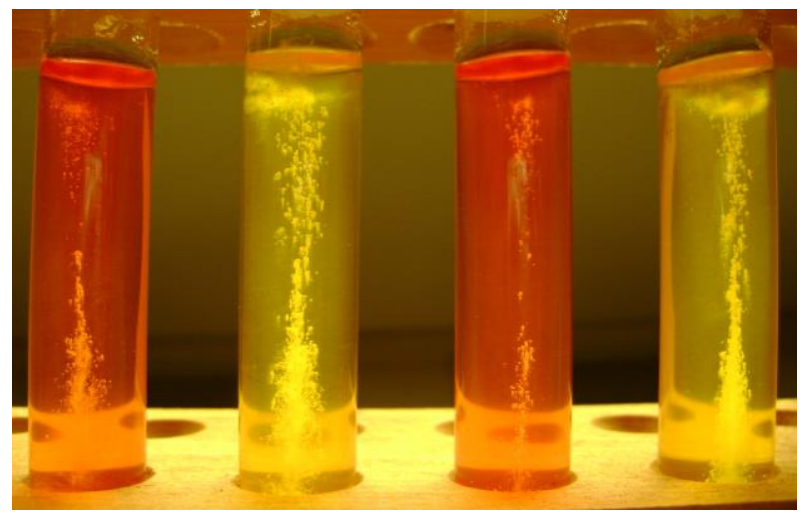

Figure 3. Clostridium acetobutylicum in phenol red broth (with $0.2 \%$ agar and Oxyrase) prepared with biodiesel byproduct. Supplements L to R: none; glucose; byproduct from column; glucose and byproduct mix (1/2 molar ratio assuming that byproduct was glycerol). Pure glycerol was not shown. Supplements were added at $0.5 \%$ concentration. 
Inoculum size was $0.1 \mathrm{ml}$ of maximum stationary phase cells in $10.0 \mathrm{ml}$ of medium. The incubation was at $25^{\circ} \mathrm{C}$ for 6 weeks.

The $\mathrm{pH}$ of cultures containing glucose dropped from 8.0 to 5.0, and the concentration of glucose dropped to an undetectable level (Table 1). This was likely to be associated with a robust culture response. Synthesis by $C$. acetobutylicum has two phases (Jones and Woods, 1986): (1.) In acidogenesis there is vegetative growth where organic acids acetate and butyrate are produced. (2.) In solventogenesis, acids produced are transformed into acetone, butanol and ethanol. In this study, the alcohol oxidase and peroxidase assay indicated the presence of alcohol in all cultures (Table 1). The Growth of $C$. acetobutylicum was very slow compared to many species of bacteria. Consequently, cultures were provided a lengthy incubation to use nutrients and synthesize alcohol. With age, some microbes, like the clostridia, make spores. It has been reported that sporulation was necessary for solvent production (Woods, 1995). Stock solutions of byproduct made by catalyst column and transesterification showed a positive test for the presence of alcohol. Therefore, It was not possible to conclude if alcohol was produced in cultures of $C$. acetobutylicum in presence of the byproduct even though culture growth was exhibited in the presence of byproduct . Byproduct produced by standard transesterification may contain residual methanol. (Samples of C. acetobutylicum cultures inoculated on BHI agar plates would not produce colonies aerobically, indicating that there was no contamination with oxygen-using facultative bacteria.)

Table 1. Biochemical characterization of Clostridium acetobutylicum cultures with biodiesel byproduct.

\begin{tabular}{lccc}
\hline Supplement & $\mathrm{pH}$ & Glucose test & Alcohol test \\
\hline None & $8.0>8.0^{2}$ & $->-$ & $->+$ \\
\hline Glucose & $8.0>5.0$ & $+>-$ & $->+$ \\
\hline $\begin{array}{l}\text { Byproduct from } \\
\text { catalyst column }\end{array}$ & $8.0>8.0$ & $->-$ & $+>+$ \\
\hline $\begin{array}{l}\text { Glucose and } \\
\text { byproduct mix }\end{array}$ & $8.0>5.0$ & $+>-$ & $+>+$ \\
\hline \begin{tabular}{l} 
Pure glycerol \\
\hline
\end{tabular} & $8.0>8.0$ & $->-$ & $->+$ \\
\hline
\end{tabular}

${ }^{\mathrm{a}}$ Uninoculated medium and after incubation of culture. + , indicated that test substrate was present. -, indicated that test substrate was absent. Incubation was at $25^{\circ} \mathrm{C}$ for 6 weeks. Reactions apply to cultures produced from fluid thioglycollate medium (with Oxyrase) and phenol red broth (with $0.2 \%$ agar and Oxyrase).

Experiments with glycerol byproduct made by catalyst column or transesterification gave results that were observed to be similar. It did not appear likely that there was any appreciable utilization of byproduct by $C$. acetobutylicum. It is possible that different strains of $C$. acetobutylicum could exhibit different biochemical phenotypes for oxidation of carbon sources and effect results. Preliminary experiments with a strain of Clostridium butyricum and byproduct showed results similar to those for the strain of $C$. acetobutylicum. Other bacterial genera may utilize the byproduct. It is possible that batches of byproduct may not all be of the same composition. For example, triacylglycerols may be from different sources and may be mixed with other kinds of waste molecules. Research should be performed to determine if the glycerol byproduct from biodiesel plants can be used to make new products as well as help solve a waste disposal problem.

\section{Acknowledgement}

This work was supported by a grant (AF31) from the Michigan Ohio University Transportation Center (MIOH UTC), and the Department of Biology at the University of Detroit Mercy.

\section{References}

Difco Laboratories (1985) Dehydrated culture media and reagents for microbiology, p. 160-164, 657-662, 951956. In The Difco Manual, 10th ed. Difco Laboratories, Detroit, MI.

Girbal L, Soucaille P (1994). Regulation of Clostridium acetobutylicum metabolism as revealed by mixedsubstrate steady-state continuous cultures: role of NADH/NAD ratio and ATP pool. J Bacteriol 176:64336438.

Jones DT, Woods DR (1986) Acetone-butanol fermentation revisited. Microbiol Rev 50:484-524.

Kalscheuer R, Stolting T, Steinbuchel A (2006) Microdiesel: Escherichia coli engineered for fuel production. Microbiology 152: 2529-2536.

Luo C, Brink D, Blanch H (2002) Identification of potential fermentation inhibitors in conversion of hybrid poplar hydrolyzate to ethanol. Biomass Bioenergy 22:125-138.

Murarka A, Dharmadi Y, Yazdani SS, Gonzalez R (2008) Fermentative utilization of glycerol by Escherichia coli and its implications for the production of fuels and chemicals. Appl Environ Microbio 74:1124-1135.

Saha BC (2004) Lignocellulose biodegradation and applications in biotechnology, p. 2-34. In Saha BC and Hayaski K (ed.) Lignocellulose Biodegradation. American Chemical Society, Washington, DC.

Vasconcelos I, Girbal L, Soucaille P (1994) Regulation of carbon and electron flow in Clostridium acetobutylicum grown in chemostat culture at neutral $\mathrm{pH}$ on mixtures of glucose and glycerol. J Bacteriol 176:1443-1450.

Woods DR (1995) The genetic engineering of microbial solvent production. TrendsBiotechnol 13:259-264. 